\section{Acesulfame: From Sugar Substitute to Wastewater Marker}

\section{Ignaz J. Buerge* and Thomas Poiger}

${ }^{*}$ Correspondence: Dr. I. J. Buerge, Agroscope Changins-Wädenswil Research Station ACW, Schloss, CH-8820 Wädenswil, Tel.: +41 4478363 83, Fax: +41 44 78063 41, E-Mail: ignaz.buerge@acw.admin.ch

\section{Keywords: Acesulfame $\cdot$ Artificial sweetener - Chemical} marker · Domestic wastewater · Groundwater

Groundwater is an important drinking-water resource and deserves a high level of protection. Contamination may originate from various sources such as agriculture, households, industry, and traffic, and knowledge on the relative contribution of these sources is necessary to take efficient protection measures.

In search of a 'perfect chemical marker' of domestic wastewater from households in groundwater, we examined artificial sweeteners as they are consumed in considerable quantities with food and beverages. Some sweeteners pass through the human metabolism largely unaffected and thus reach the environment associated with domestic wastewater. One of these sweeteners, the sulfoamide acesulfame, was found ubiquitously in wastewater, surface waters, and groundwater from Switzerland.

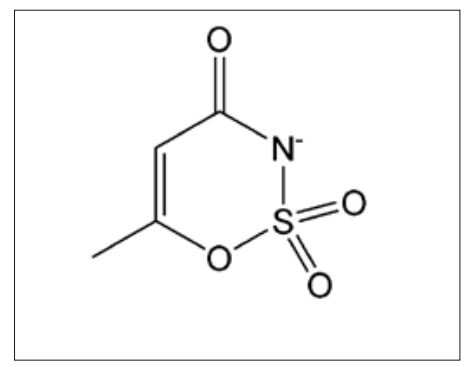

Chemical structure of the artificial sweetener acesulfame. The compound is negatively charged at environmental $\mathrm{pH}$ values and thus very mobile.
Concentrations of acesulfame in wastewater ranged from 12 to $46 \mu \mathrm{g} / \mathrm{L}$. The compound was not eliminated in wastewater treatment plants. Consequently, acesulfame was also detected in most surface waters, where concentrations correlated nicely with the actual wastewater burden. Furthermore, acesulfame was detected in $65 \%$ of the investigated groundwater samples. Thereby, the highest concentrations (up to $4.7 \mu \mathrm{g} / \mathrm{L}$ ) were observed in areas where significant infiltration of river water with a high wastewater burden occurs. At certain pumping stations, our data suggest that up to $20 \%$ of the water indirectly stems from domestic wastewater. For drinking water production, groundwater is often not further purified in Switzerland, and acesulfame was thus also found in several tap water samples (up to $2.6 \mu \mathrm{g} / \mathrm{L})$. However, acesulfame is susceptible to ozonation (elimination, $\approx 85-95 \%$ ).

The sweetener is analyzed using a $\mathrm{C}_{18}$ reversed phase column and a triple quadrupole mass spectrometer operated in multiple reaction monitoring mode. As no off-line preconcentration step is necessary, results are obtained within $1 \mathrm{~h}$ or less.

Acesulfame thus meets all criteria for a 'perfect' marker of domestic wastewater in groundwater. It is source-specific, reflects contamination in a quantitative sense, and is amenable to rapid and sensitive analysis by liquid chromatography-tandem mass spectrometry. Given the currently achieved detection limit of $\approx 0.01 \mu \mathrm{g} / \mathrm{L}$, it is possible to trace the presence of $\geq \mathbf{0 . 0 5 \%}$ wastewater in natural waters. Finally, acesulfame is sufficiently persistent and hydrophilic to reach groundwater.

Received: January 14, 2011

References

I. J. Buerge, H. R. Buser, M. Kahle, M. D. Müller, T. Poiger, Environ. Sci. Technol. 2009, 43, 4381.

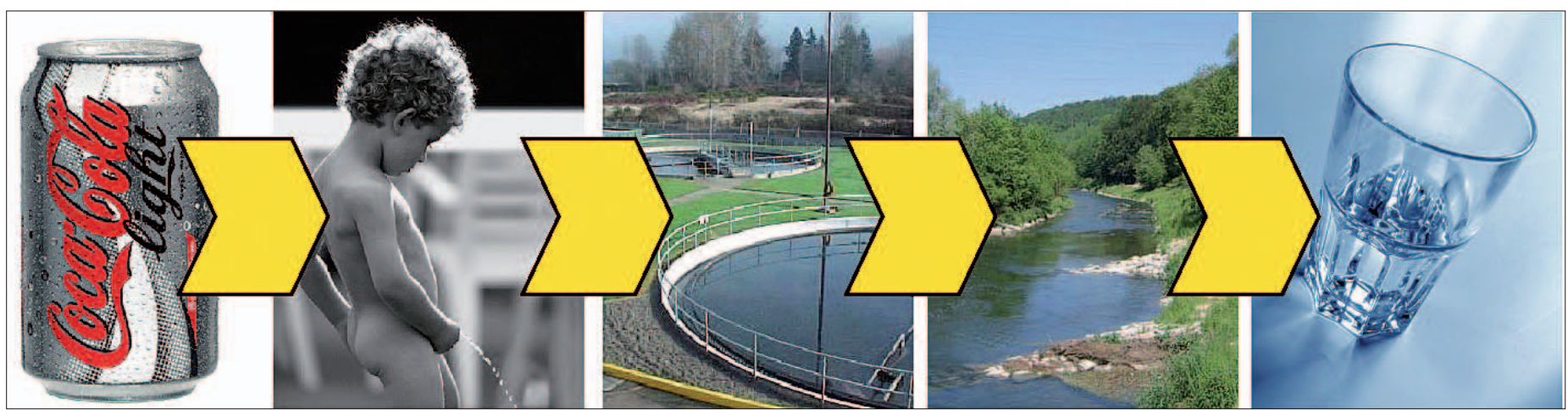

The fate of acesulfame from its consumption as sugar substitute in light-beverages to renal excretion, passage through wastewater treatment plants, discharge to surface waters, infiltration into groundwater, and detection in tap water. Second photo from the left: 1 art1 $\mathrm{GmbH}$ 